\title{
V. On central forces and the conservation of energy
}

\section{Walter R. Browne M.A. M.Inst.C.E}

To cite this article: Walter R. Browne M.A. M.Inst.C.E (1883) V. On central forces and the conservation of energy, Philosophical Magazine Series 5, 15:91, 35-42, DOI: 10.1080/14786448308627304

To link to this article: http://dx.doi.org/10.1080/14786448308627304

$$
\text { 曲 Published online: } 28 \text { Apr } 2009 .
$$

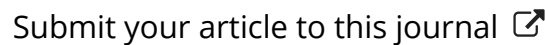

Џ Article views: 2

Q View related articles 5 
In its peculiar sensitiveness to the visible part of the spectrum selenium seems, so far as our present knowledge goes, to stand almost, if not quite, alone*.

Riverstone Lodge, Southfields,

Wandsworth, S.W.

V. On Central Forces and the Conservation of Energy. By Walter R. Browne, M.A., M. Inst. C.E, late Fellow of Trinity College, Cambridge $\dagger$.

$\mathrm{T} T$ is well known that the ordinary proof of the principle 1 known as the Conservation of Energy assumes the forces acting to be Central Forcesł; but the intimate connexion existing between these two facts-the existence of Central Forces and the Conservation of Energy-has not, so far as $I$ am aware, been thoroughly examined. I shall here attempt to show that the two necessarily imply each other; so that not only is the Conservation of Energy true if the system is a system of central forces, but the Conservation of Energy is not true if the system is any thing but a system of central forces.

For the sake of simplicity I will confine myself to the case of two particles, and suppose them so far apart, in proportion to their dimensions, that each may be treated as if concentrated at its centre of gravity. Let the particles be $A$ and $B$, and consider the motion of $B$ with reference to $A$ as fixed. Suppose $B$ to be moving away from $A$, and to be acted upon by a moving force due to the action of A. Let it move from a distance $a$ to a distance $a+b$, and let $\mathrm{F}$ be the resolved part of the moving force in the line $\mathrm{AB}$. Then the energy exerted by $B$ during this motion in overcoming the attraction of $A$ is represented by

$$
\int_{a}^{a+b} \mathrm{~F} d x .
$$

Let $v_{1}$ be B's initial velocity, $m$ its mass. Then at the end of the motion $v_{1}$ will be reduced to $v$, where $v$ is given by the

* So far as regards Dr. Moser's application of his theory to the carbon photophone of Messrs Bell and Tainter, I entirely agree with him; my own experiments showing conclusively that the effects are due to heat only. But the best carbon cells are vastly inferior in their action to those of selenium.

$\dagger$ Communicated by the Physical Society, having been read at the Meeting on November 11, 1882.

t This is recognized explicitly by Clausius, 'Mechanical Theory of Heat,' p. 16. 
equation

$$
\frac{m}{2}\left(v_{1}^{2}-v^{2}\right)=\int_{a}^{a+b} \mathrm{~F} d x .
$$

Let us suppose $b$ to be such that $v=0$, so that

$$
\frac{m}{2} v_{1}^{2}=\int_{a}^{a+b} \mathrm{~F} d x . . . . . . .
$$

Then, when $\mathrm{B}$ arrives at distance $\overline{a+b}$, its velocity, and therefore its kinetic energy or vis viva, will be reduced to zero. There is therefore a loss of energy, so far as B is concerned. But now let us suppose that $B$ is left free to return towards A, and that it passes back again over the space $b$. Then, if $\mathrm{F}$ continues to act, $A$ will exert during the motion an amount of energy on $B$, or will do an amount of work upon $B$, which will be represented by

$$
\int_{a+b}^{a} \mathrm{~F} d x
$$

and when $\mathrm{B}$ has reached the distance $a$, it will have gained $\mathrm{a}$ velocity $\mathrm{V}$, given by the equation

$$
\frac{m}{2} \mathrm{~V}^{2}=\int_{a+b}^{a} \mathrm{~F} d x . \quad . \quad . \quad . \quad . \quad .
$$

Now if $\mathrm{V}=-v_{1}$, then $\mathrm{V}^{2}=v_{1}^{2}$ : hence we shall have the two particles in the same position as at first, and the kinetic energy of $B$ will be the same as at first. Therefore there will have been no loss or gain of energy on the whole; and the energy is then said to have been conserved during the motion. At the time when B's velocity is zero, the energy of the system is represented by the potential energy of A-that is, the power $\mathrm{A}$ has of subsequently doing the work $\int_{a+b}^{a} \mathrm{~F} d x$ upon $\mathrm{B}$. At other times during the motion, the energy of the system is partly potential energy of $A$, partly kinetic energy of $B$.

We thus see that it is essential to the Conservation of Energy that $\mathrm{V}^{2}$ should $=v_{1}{ }^{2}$. But by equations (1) and (2) this is equivalent to the equation

$$
\int_{a}^{a+b} \mathrm{~F} d x=\int_{a \vdash b}^{a} \mathrm{~F} d x ; \ldots
$$

these two expressions representing the two amounts of energy exerted, as described above.

It is therefore essential for the conservation of energy that 
$F$ should be a function of a form such that equation (3) may hold. We have now to inquire what this form must be, or, in other words, within what limits $\mathbf{F}$ may be allowed to vary so that the equation (3) may still hold.

Now we have not supposed the constitution of $A$ and $B$, or their relations to each other, to vary in any way except in regard to space and in regard to time; and we have every reason to believe that these are the only variations which take place in the ultimate molecules of matter. Hence we need only consider variations with regard to space and time.

Now if $\mathrm{F}$ be any function of time, then, since some time must have elapsed between the exertion of the two amounts of energy represented by the two sides of equation (3), it follows that for every value of $\mathrm{F}$ in the right-hand expression the time will be greater than for the corresponding value of $\mathrm{F}$ in the left-hand expression; and therefore the sums of the two sets of values, or the two integrals, cannot be equal. Hence $\mathrm{F}$ cannot be a function of time.

We have therefore only to consider variations in space. Now, if we confine our attention to one plane, we know that any variation of $B$ 's place in that plane may be represented by a change in the values of $x$ and $\theta$; where $x$ is $\mathrm{B}$ 's distance from A, and $\theta$ the angle which the axis of $x$ makes with some fixed line in the plane*. Then it is easy to show that $\mathrm{F}$ must not vary with $\theta$. For if it does, let us suppose that when $B$ has come to rest, and before it is allowed to return, it is made to rotate about $A$ through an angle $d \theta$, and again brought to rest. Then the circumstances of $A$ and $B$ are unchanged; for the kinetic energy given to B during the rotation has been taken out again in stopping it. But if $B$ is now allowed to return towards $A$, then, for every value of $F$ in the right-hand expression, the value of $\theta$ will be greater or less by $d \theta$, the amount of the change, than for the corresponding value in the left-hand expression; and therefore, as before, the two integrals cannot be equal. Similarly, if we take coordinates $x, \theta, \phi$, in three dimensions, it will follow that $F$ cannot be a function of $\phi$.

Hence we are left with the conclusion that $\mathrm{F}$ can only be a function of $r$; in other words, the force with which $A$ acts upon $B$ always tends towards $A$, and varies, if it varies at all, according to the distance from $A$ only. But this is the definition of a central force.

[The proof just given, that $\mathrm{F}$ cannot vary with $\theta$, appears

* We here make no assumption except that the force varies as B's position in the plane varies; which is essential to every theory on the subject. 
quite general. But it is easy to show that any particular law of force which can be imagined, other than that of a central force, is inconsistent with the conservation of energy. Thus, suppose the force to vary according to B's distance from some other point in the plane than $A$; then that distance cun always be expressed in terms of the coordinates of its extremities, and therefore in an expression involving $\theta$, which is inadmissible. Again, suppose the force to vary according to the perpendicular distance of $B$ from some line in the plane. Then, if B move parallel to that line the force is constant, while if it be perpendicular it varies from zero; and it is easy to see that if $B$ moves perpendicular to that line, and if, before it is allowed to return, it is rotated till the line $A B$ is parallel to that line, then the two integrals will not be equal. Again, suppose the force to act upon a certain line only, so that when $B$ is off that line no force acts upon it; then, if we suppose the return journey made parallel to that line, the energy on that journey is zero.]

We have throughout taken $\mathrm{F}$ as the force between $\mathrm{A}$ and $\mathrm{B}$, resolved along the line joining them. We have still to consider the possibility of there being another component always at right angles to this line. This component, if it exists, will produce a rotation of $B$ round $A$; which will increase $B$ 's kinetic energy; and as there will be nothing to balance it, this increase will go on for ever; so that the conservation of energy would not be true in this case.

I have thus proved, I believe, the proposition with which I started-namely, that the doctrine of central forces and that of the conservation of energy are mutually interdependent, so that one is not true without the other. In general, as remarked at the beginning, the existence of central forces is assumed, and the conservation of energy deduced from it. But the process may be reversed. The conservation of energy may be considered to rest, as a general law of nature, on the broad basis of observed facts, such as the conversion of heat, electricity, chemical actions, \&c. into mechanical work, and the reconversion of mechanical work into these other forms of energy. There can be no doubt that the evidence of this character is of very great weight; and I am myself disposed to accept it as conclusive. But it must be pointed out that, unless the above investigation be false, it involves our accepting a mechanical definition of matter substantially to the following effect*:- "Matter consists of a collection of centres

* This definition has been already given in a pamphlet entitled "The Foundations of Mechanics" (Charles Griffin and Co., 1882). 
of force, acting upon each other according to laws which do not vary with time but do vary with distance."

This conception of matter is of course an old one, being that usually known by the name of Boscovitch. It has not, however, been generally accepted by writers on Mechanics; and in recent times certain special objections have been raised against it, which it seems well briefly to consider.

1. An objection, due to Professor Maxwell*, is that the conception does not comprise the idea of inertia, which is a fundamental fact with regard to matter. But when we say that a body has inertia, we simply mean that a finite force, acting upon it for a finite time, generates only a finite velocity. Hence it follows that any body we can see or feel, or know and investigate in any way, must have inertia; for suppose a body to possess no inertia, then the first time any force was applied to it, it would at once be removed to an indefinite distance, and would therefore be beyond the reach of investigation. To say that matter has inertia is therefore merely to assert the general principle that any thing our senses can deal with must be finite; and it is therefore a condition anterior to any theory of matter, not a part of such theory.

2. An objection given by Lamé $\dagger$ is that bodies, and especially homogeneous crystals, are not, within the limits of observation, denser at the centre than they are at the surface, which on the theory of central forces they apparently should be. But on this it may be observed as follows.

It may be admitted that collections of centres of force, at rest under their mutual actions, would be more dense towards the centre. We know no such bodies in nature. The nearest approach to it is the case of bodies so large that their molecular motions, and also their want of homogeneity, may be neglected in comparison of their mass. But the condition of large bodies does appear to agree with the theory; e.g. even the mean specific gravity of the earth $(5 \cdot 6)$ is greater than that of all bodies, except a few metals, at the surface. The want of homogeneity can have little influence at the temperature and pressure which prevail in the interior.

Again, it is known that, in all bodies, the actual centres of force must be bound up together in molecules so closely as to form coherent wholes, which no known force can change or break up. The relations in a crystal therefore are not those among separate centres of force, but among separate molecules.

Again, these molecules, being hot, are in rapid and continuous motion.

* 'Theory of Heat,' p. 85.

† Eltaticité des Corps solides, p. 333. 
Lastly, the laws of the forces of cohesion, whether in the interior of a molecule or between one molecule and another, are unknown.

In such circumstances, can it be held impossible that there should be laws of distribution of force such that in small bodies like crystals the difference in density at the centre and surface should be insensible? Lamé does not attempt to give any rigid proof that the uniform density of crystals (even if accurately true) is really incompatible with the theory of central forces. It is therefore merely a presumption, and a presumption which seems seriously weakened by the foregoing considerations; it cannot therefore be allowed to have any weight as against actual evidence.

3. An objection, due to Prof. Tait, is that we have no right to assume that force has any objective existence at all, or is any thing more than the rate of change of motion-and that in fact it cannot have an objective existence, because it can be affected with a positive or negative sign. But, with regard to the first part of this objection, a force is defined in Mechanics simply as a cause of motion; and therefore the remark is a mere denial of the general principle of causation. This is not the place to discuss the truth of that principle; but it may be observed that it is perhaps almost the only principle which may claim to have been accepted by all thinkers of all schools and in all ages. With regard to the second part of the objection, the circumstance that a force, or rather the symbol of a force, may be affected, for purposes of calculation, with a + or - sign is simply due to the fact that a force has a definite sense, or direction; and that direction is one of the properties of things to which the conception of positive and negative may properly be applied. For the same reason lines may be represented as + or - , as in algebraical geometry; but they are not therefore regarded as non-existent. Nor is direction the only fact to which the conception applies; e. $g$. in treatises on algebra it is often pointed out that capital may be taken as positive and debt as negative. Will it therefore be argued that money has no real existence?

4. In some quarters an objection appears to be felt to the theory of central forces, on the ground that it involves the conception of action at a distance, which is supposed to be " unthinkable." I am not aware that the term "unthinkable," which is a new one, has ever been defined. Until it has been, it is impossible to say whether action at a distance is unthinkable, or whether the fact of a conception being unthinkable is sufficient reason, or any reason, for holding it to be untrue. 
It seems desirable, before leaving the subject, to say a few words upon a theory which has been set up as a rival to that of central forces, and in some quarters has met with considerable favour. This theory supposes that bodies can act on each other only when in absolute contact; and that all the phenomena of the universe may be accounted for by the knockings together of a number of ultimate atoms, considered as very small impenetrable bodies, moving with high velocities in space.

It might be urged that before such a theory can be seriously discussed, it must be shown capable of explaining (as the theory of central forces certainly does explain) the facts and principles of Mechanics. I am not aware that this has been done. I may, however, point ont that the theory is not inconsistent with the conservation of energy ; that is to say, it can be reconciled with it by certain special assumptions. For the proof of that principle, as given above, does not necessarily imply that the forces acting are continuous. If the attraction of $A$ be supposed to act on $B$ by equal impulses at certain intervals of space, or distances from $A$, which distances remain always the same, then the proof will still hold; for B will be acted upon by exactly the same number of impulses, and at exactly the same places, on its return journey as on its outward journey, and the effects will therefore be the same. Now the "collision" theory above mentioned may be taken to represent the extremest possible case of this discontinuous actionthere being then but one impulse, and that acting when $A$ and $B$ are in absolute contact.

Let us, however, consider the assumptions involved, if the conservation of energy is to hold in this extreme case. Imagine two " ultimate atoms," of equal mass, to meet each other with equal velocities in the same straight line. This is clearly a possible case under the theory; and the conservation of energy must therefore be consistent with it. Then the instant before the atoms meet they have no action upon each other, and the instant after, by symmetry, they must either be at rest or must have passed through one another. As the latter is contrary to the hypothesis, they must be at rest. Hence a finite mass moving with a finite velocity has been brought to rest in a space infinitely small; and therefore the impulse acting upon it must have been strictly infinite in amount. This collision therefore (and it is easily seen that the same will be true of all collisions) occasions the instantaneous development of a strictly infinite force. The atoms being brought to rest, there is no reason to be given why any thing further should happen. But we must assume it as an axiom that a further mutual im- 
pulse is then given, sufficient (if the bodies are supposed perfectly elastic) to cause each to return on its path with a velocity exactly equal to that with which it arrived. This further impulse must also be instantaneous and infinite; for, force being the cause of motion, if the impulse were finite it would at once cause the bodies to separate through an indefinitely small space, and then, ex hyp., no further action could take place, and the bodies would recede from each other with indefinitely small velocities. If, then, we make these three assumptions-(1) that there is an infinite impulse developed on the collision, which brings the atoms to rest, (2) that there is a further infinite impulse, which separates them, (3) that this further impulse, while infinite, is such as exactly to reverse the previous motion of each particle - then the conservation of energy may still be supposed to hold through the collision.

It remains to ask whether there are any advantages in the collision theory such as would warrant us in discarding the principle of continuity, and in making the somewhat violent assumptions described above. The advantages specially claimed by its advocates appear to be that it does away with the conception of action at a distance, and also with that of potential energy. The latter supposition, however, is not correct. At the instant when the two atoms are at rest their actual energy is zero, and the energy existing is entirely potential, being due to their capacity of generating a return velocity equal to that of arrival. Of the former supposition I have already spoken; and I may add that I have elsewhere* shown it to be impossible to explain certain elementary facts of physics without the hypothesis of action at a distance.

\section{Relations between the Heats of Combination of the Elements and their Atomic Weights.}

To the Editors of the Philosophical Magazine. Gentlemen,

THE periodic variation of the properties of the elements 1 with their atomic weights is now accepted as proved by chemists; but no similar connexion seems yet to have been established between the atomic weights and heats of combination of the elements. Now, if the atomic weights of the elements are taken as abscissæ, and their atomic heats of combination with chlorine as ordinates of a curve, the heats of combination will be seen to be a periodic function of the atomic weights.

* “Un Action at a Distance," Phys. Soc. 1881; Phil. Mag. Dec. 1880. 